\title{
Central Nervous System Necrotic Lesion
}

National Cancer Institute

\section{Source}

National Cancer Institute. Central Nervous System Necrotic Lesion. NCI Thesaurus. Code C78236.

A necrotic process affecting the brain and/or spinal cord. 